\title{
Population synthesis of pulsars: Magnetic field effects
}

\author{
T. Regimbau and J. A. de Freitas Pacheco
}

\author{
Observatoire de la Côte d'Azur, BP 4229, 06304 Nice Cedex 4, France \\ e-mail: pacheco@obs-nice.fr
}

Received 1 September 2000 / Accepted 26 April 2001

\begin{abstract}
New results based on methods of population synthesis, concerning magnetic field effects on the evolution of pulsars, are reported. The present study confirms that models with timescales for the magnetic field decay longer than the pulsar lifetime are in better agreement with the data. These new simulations indicate that the diagram $\log (P \dot{P})-\log \left(t_{\mathrm{s}}\right)$ alone cannot be used to test field decay models. The dispersion of the values of the initial period and magnetic field can explain the observed behaviour of the data points in such a diagram. The simulations also indicate that the statistical properties of anomalous X-ray pulsars and soft-gamma repeaters (magnetar candidates) are compatible with those derived for objects born in the high side tail of the magnetic field distribution. The predicted birth rate of neutron stars having field strengths in excess of $10^{14} \mathrm{G}$ is one object born each $750 \mathrm{yr}$.
\end{abstract}

Key words. pulsars - population synthesis - magnetars

\section{Introduction}

The magnetic field is probably one of the most important parameters affecting the evolution of a pulsar. The field strength fixes the energy loss rate, the luminosity in different spectral regions and thus, the observability of pulsars. However, since the very first studies on the field evolution (Gunn \& Ostriker 1970), the subject has remained highly controversial. The original statistical study by Gunn \& Ostriker, based on a small number of objects, led to the conclusion that the magnetic field decays on time scales of few Myr, due to Ohmic dissipation. The analysis of proper motions by Lyne et al. (1982) seems to support that conclusion (see also Narayan \& Ostriker 1990), but numerical simulations by Bhattacharya et al. (1992), Hartman et al. (1997) and Mukherjee \& Kembhavi (1997) suggest that the observed properties of the pulsar population are consistent with decay times longer than the pulsar lifetime.

Over the past years there has been increasing recognition that neutron stars are born with a considerable diversity of magnetic field strengths and of initial rotation periods. In particular, Thompson \& Duncan (1995) suggested the existence of neutron stars, dubbed "magnetars", with magnetic field strengths in excess of $10^{14} \mathrm{G}$. Observations of soft gamma-ray repeaters and anomalous X-ray pulsars (Kouveliotou et al. 1994, 1998, 1999; Cline et al. 2000; Vasisht \& Gotthelf 1997; Mereghetti 1999) give some support to the assumption that these objects could be associated with highly magnetized neutron stars, if the

Send offprint requests to: T. Regimbau,

e-mail: regimbau@obs-nice.fr braking mechanism is the "standard" magnetic-dipole radiation. It is worth mentioning that if the star also loses angular momentum by accelerating a flow of relativistic particles, then the derived magnetic field should have smaller strengths in comparison with the values obtained from the canonical model. These sources have typical X-ray luminosities in the range $L_{\mathrm{X}} \approx 10^{35-36} \mathrm{erg} / \mathrm{s}$ and surprisingly, these values are greater than the expected magnetic dipole luminosity $I \Omega \dot{\Omega}$ for most of the objects. A magnetic origin for the X-ray emission was considered by Thompson \& Duncan (1996) and Heyl \& Kulkarni (1998), among others, and possible magnetic field decay paths as well as their respective time scale were reviewed by Goldreich \& Reisenegger (1992). If magnetic field dissipation is indeed the energy source of this emission, then typical decay time scales are of the order of $10^{4} \mathrm{yr}$ (Colpi et al. 2000).

More recently, Urpin \& Konenkov (1997) studied the magnetic field evolution of neutron stars, under the assumption that the field is initially confined to the crust. According to their calculations, the magnetic field evolution depends on the conductive properties of the crust, which are linked to the thermal history of the neutron star. Their model computations suggest that the field decays more slowly than the classical exponential law resulting from pure Ohmic dissipation. They have revisited the diagram originally employed by Gunn \& Ostriker (1970), in which the quantity $P \dot{P}$ is plotted against $t_{\mathrm{s}}=P /(2 \dot{P})$. In the standard magnetic dipole model, the former is related to the square of the magnetic field strength whereas the latter, to the pulsar age. Urpin \& Konenkov interpret this 
plot as an indication that the magnetic field decays, but recognize that the observed trend may be caused by the wide range of the period derivative values. In fact, Lyne et al. (1975) conjectured that the observed trend in the plane $P \dot{P}-t_{\mathrm{s}}$ could be explained by considering only the dispersion in the observed quantities $P$ and $\dot{P}$.

In a previous paper (Regimbau \& de Freitas Pacheco 2000, hereafter Paper I), statistical properties of pulsars derived from population synthesis based on Monte Carlo methods were reported, and the derived properties of the "unseen" objects were used to study their contribution to the continuous gravitational wave emission in the galactic disk. In this work, we present results of new simulations, using the up-graded code addressed to the following questions: i) is the field decay time scale longer than the pulsar lifetime, as suggested by other simulations? ii) are the anomalous X-ray pulsars (AXP) and the soft gammaray repeaters (SGR) a distinct class of neutron stars? In other words, if a high magnetic field is responsible for the observed characteristics, are these objects simply associated with neutron stars born in the high side tail of the field distribution? iii) Can the observed trend in the plane $P \dot{P}-t_{\mathrm{s}}$ be explained only by dispersion of the initial values of the rotation period and magnetic field? The plan of this paper is the following: in Sect. 2 we describe briefly the model on which the numerical code is based, in Sect. 3 we report the main results of this work and finally, in Sect. 4 we discuss and present our main conclusions.

\section{The model}

A detailed presentation of our model was already reported in Paper I and here, for the sake of completeness, only the principal aspects are reviewed.

In our code, pulsars are generated at a constant rate with initial periods and magnetic fields distributed according to Gaussian and log-normal probability functions respectively. Their initial positions in the Galaxy are established by assuming exponential distributions, having a scale of height of $100 \mathrm{pc}$ along the $z$-axis and $2.3 \mathrm{kpc}$ along the radial direction. A scenario excluding recent pulsar formation in the inner galactic bulge $(1 \mathrm{kpc}$ around the galactic center) was also considered, but the final results are not considerably affected by this assumption. The effect of an initial random kick velocity on the orbital motion is also taken into account. In our simulations, the transversal and radial (coincident with the direction of the orbital motion) components of the kick velocity are assumed to have a Gaussian distribution (Lorimer 1993) with velocity dispersions of $140 \mathrm{~km} \mathrm{~s}^{-1}$ and $100 \mathrm{~km} \mathrm{~s}^{-1}$ respectively, corresponding to an average kick velocity of $170 \mathrm{~km} \mathrm{~s}^{-1}$. Simulations with average velocities of $300 \mathrm{~km} \mathrm{~s}^{-1}$ (Hansen \& Phinney 1997) and $450 \mathrm{~km} \mathrm{~s}^{-1}$ (Lorimer \& Lyne 1994) were also performed without any appreciable modification in the results. Simulations with high kick velocities produce a significant number of objects which escape from the Galaxy, modifying slightly the pulsar birthrate.
In order to model the evolution of the rotation period, two scenarios were considered. In the first, the standard magnetic dipole model was modified to permit a continuous increase of the angle between the spin and the magnetic dipole axes, but keeping constant the field intensity (model A). In the second, the magnetic field was allowed to decay exponentially (model B). The migration of the magnetic dipole from an initial arbitrary angle to an orthogonal position with respect to the spin axis is a consequence of the scenario explored by Link et al. (1998) and Epstein \& Link (2000), where starquakes in spinningdown pulsars may push matter toward the poles, causing an increasing misalignment of those axes. This possibility is quite attractive, since during the migration phase, braking indices smaller than three, as found in some young pulsars, can be obtained (see Paper I) and it could also be a possible explanation for the increasing spin-down rates observed in some pulsars and SGRs, as we shall see later. In this case, the period evolution is given by

$$
P=P_{0}\left[1+\frac{t}{\tau_{0}}-n_{0} \frac{t_{\alpha}}{\tau_{0}}\left(1-\mathrm{e}^{-t / t_{\alpha}}\right)\right]^{1 / 2}
$$

where $P_{0}$ is the initial period, $\tau_{0}=\frac{3}{4 \pi^{2}} \frac{I c^{3} P_{0}^{2}}{B^{2} R^{6}}$ is the magnetic braking time scale and $t_{\alpha}$ is the magnetic dipole migration time scale. The parameter $n_{0}$ corresponds to the initial angle $\alpha$ between the spin and the magnetic axes such as $\alpha=\operatorname{arcos}\left(\sqrt{ } n_{0}\right)$. Tauris \& Manchester (1998), from the analysis of polarization data, found a tendency for the magnetic axis to align with the rotational axis on a timescale of about $10^{7} \mathrm{yr}$. In fact, they assumed a time evolution for the angle $\alpha$ of the same form as that suggested by Jones (1976), namely,

$\sin \alpha(t)=\sin \alpha_{\mathrm{o}} \mathrm{e}^{-t / t_{\mathrm{a}}}$.

In this situation, the solution for the period evolution is formally identical that obtained when one assumes an exponential decay for the magnetic field (case B). Thus, the results of case B (discussed below) concern also the scenario envisaged by Tauris \& Manchester (1998). It should be emphasized, as those authors did, that such a scenario implies a decreasing magnetic torque and, as a consequence, a braking index greater than three, in disagreement with observations.

The detectability of pulsars is affected by: a) the fact that their radio emission is not isotropic, b) pulse broadening due to instrumental effects and c) dispersion and scattering through the interstellar medium. All these effects are included in our simulations (see Paper I for details).

Our data are a culled sample of 491 pulsars extracted from the up-graded catalog of Taylor et al. (1993), where only single objects supposed to have originated from population I stars and with all required parameters measured were considered (The word "single" here does not exclude the possibility that the progenitor evolved in a binary system, which was disrupted at the moment of the explosion, imparting momentum to the newly born neutron star). Pulsars not included in any of the original surveys 
were also discarded. Since this catalog is a compilation of different surveys covering specific sky areas and having a well defined sensitivity, the objects were distributed in sub-classes, according to the original surveys in which they were first detected. In the present study, pulsars detected by the $76 \mathrm{~m}$ radio telescope at Jodrell Bank were also included in our sample. The main characteristics of this instrument to be added to Table 1 of Paper I are: $T_{\mathrm{r}}=50 \mathrm{~K}, \tau_{\mathrm{sam}}=0.6 \mathrm{~ms}, C_{\mathrm{DM}}=0.016 \mathrm{~ms} \mathrm{~cm}^{3} \mathrm{pc}^{-1}$ and $A_{0}=0.19 \mathrm{mJy} / \mathrm{K}$.

For each pulsar generated, our code follows its evolution in order to compute the present $P, \dot{P}$ values and galactic coordinates defining the survey (or surveys) included in the general catalog, that covered such a region of the sky. The next step is the application of "filters" defining the detectability of the object, as mentioned above. Our procedure insures that the simulated catalog will have objects detected by different surveys distributed in the same proportion as in the global catalog. The numerical experiments generate objects according to these prescriptions until the simulated sample be equal to the data sample. For a given run, defined by a set of initial parameters, the number of experiments is comparable to the number of objects in the actual data sample and the final result is a suitable average, in order to avoid statistical fluctuations. For each experiment so defined, we compare the resulting distributions with those derived from present data. The values of the input parameters were optimized, by controlling the fit quality through $\chi^{2}$ and Kolmogorov-Smirnov tests.

\section{Results}

Until recently, the general belief was that most pulsars were born with parameters similar to those of the youngest known objects: the Crab $(P=33 \mathrm{~ms})$ and PSR 0540-69 $(P=50 \mathrm{~ms})$ are well known examples. Different observational facts suggest that most pulsars are born with periods $P \geq 100 \mathrm{~ms}$ (see, for instance, Bhattacharya 1990). Indeed, fifteen young pulsars with ages less than $50 \mathrm{Kyr}$ listed in the updated Taylor et al. (1993) catalog have an average period equal to $200 \mathrm{~ms}$.

The results of the present simulations compared with a data sample larger than that used in Paper I, confirm that the average initial rotation period of pulsars is $\left.<P_{0}\right\rangle=290 \mathrm{~ms}$ with a dispersion of $100 \mathrm{~ms}$ (model A). It should be emphasized that these values give the best representation of the observed period distribution even when the magnetic field decay is included (model B). In Fig. 1 the simulated period distribution for both scenarios is compared with the present data and the optimized input parameters for these models are given in Table 1. In columns two and three, the parameters defining the initial (Gaussian) distribution of periods and (log-normal) magnetic field braking time scale (average and dispersion values) are respectively given; in columns four and five are respectively indicated the pulsar lifetime and the magnetic field decay time scale. Notice that model B requires
Table 1. Optimized model parameters.

\begin{tabular}{lcccc}
\hline Model & $P_{0} \pm \sigma_{P_{0}}(\mathrm{~ms})$ & $\ln \left(\tau_{0}\right) \pm \sigma_{\ln \left(\tau_{0}\right)}$ & $t_{\max }(\mathrm{Myr})$ & $t_{\mathrm{D}}(\mathrm{Myr})$ \\
\hline $\mathrm{A}$ & $290 \pm 100$ & $9.0 \pm 3.6$ & 25 & $\infty$ \\
$\mathrm{B}$ & $290 \pm 100$ & $10.6 \pm 2.6$ & 25 & 15 \\
\hline
\end{tabular}

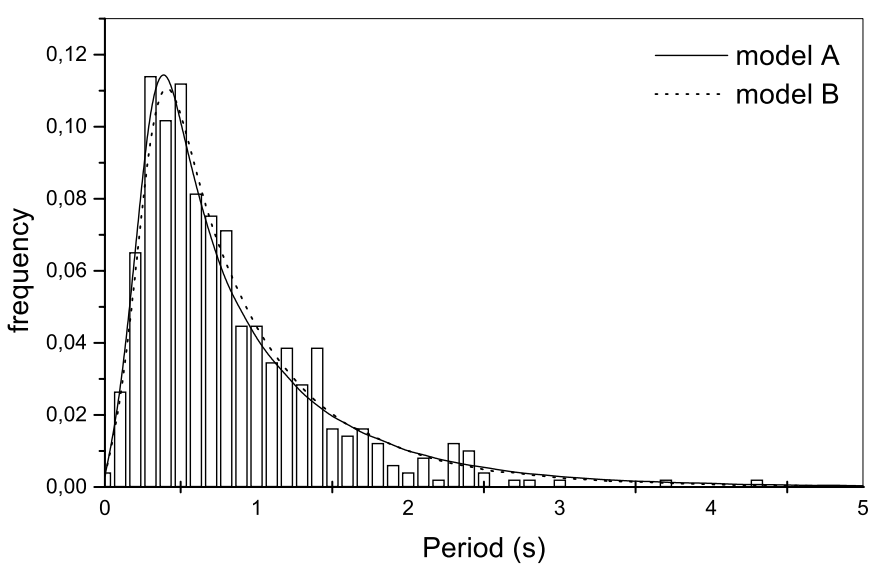

Fig. 1. Comparison between simulated period distributions for models A and B (continuous and dashed curves) and data (histogram). The simulated distributions correspond to the optimized parameters listed in Table 1.

a higher magnetic braking time scale $\tau_{0}$ and a smaller dispersion of this quantity. This is a consequence of the decreasing magnetic torque due to the field decay, requiring a longer time interval to produce the same deceleration. Concerning the dipole migration time scale $t_{\alpha}$, the new simulations simply confirm the conclusions of Paper I. It is worth mentioning that three magnetar candidates 1E 1048-5937 (Mereghetti 1995), SGR 1900+14 (Kouveliotou et al. 1999) and 1RX J1708-40 (Kaspi et al. 2000 ), with indicative ages $t_{\mathrm{s}}<t_{\alpha} \approx 10^{4}$ yr (see Table 2 below), have undergone episodes in which the spin-down rate has almost doubled. The resulting enhanced magnetic torque is consistent with the migration scenario originally developed by Link et al. (1998).

However, the situation is completely different concerning the period derivative distribution. The inclusion of the field decay displaces the maximum of the distribution toward lower values, since the decreasing magnetic torque weakens the pulsar deceleration, producing more objects with smaller period derivatives. Figure 2 compares the simulated period derivative distribution derived for models A and B with actual data where the mentioned effect can clearly be seen. If the initial parameters of model B are modified, in particular the magnetic field, in order to improve the fit quality, then a good representation of the period distribution is no longer obtained. Hartman et al. (1997) had similar difficulties in their simulations, since they were not able to simultaneously reproduce the distributions of periods and magnetic fields using models with short decay time scales.

The plane $P \dot{P}-t_{\mathrm{s}}$, which is equivalent to a plot of the square of the magnetic field versus the indicative age 


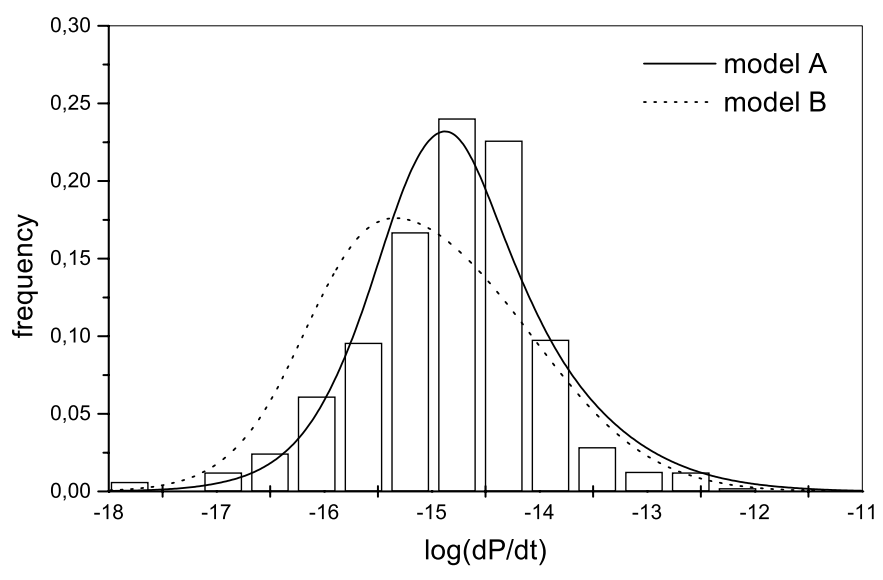

Fig. 2. Same as Fig. 1, but for the period derivative distribution.

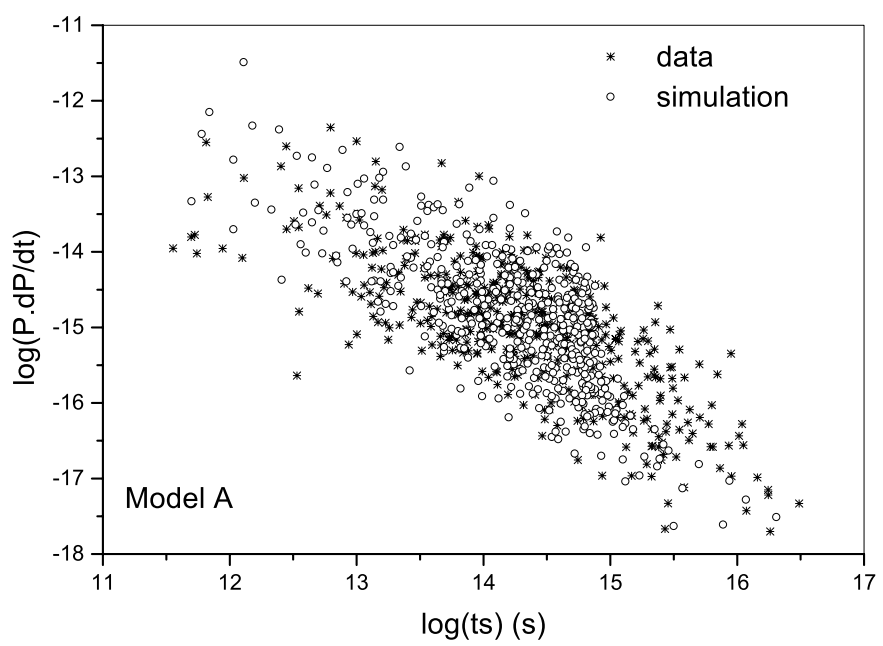

Fig. 3. Comparison between simulated (model A) and actual data in the plane $\log (P \dot{P})$ vs. $\log \left(t_{\mathrm{s}}\right)$. Simulated points have the mean slope and scattering amplitude comparable to actual data.

of the pulsar, has been used by several authors to test effects of the field decay (Gunn \& Ostriker 1970; Urpin \& Konenkov 1997). Our simulations indicate that even without including magnetic dissipation, the observed data trend on that plane can be reproduced quite well (Fig. 3). Model B does not improve the results and, in fact, a combined statistical test of the distribution of points along both axes favors model $\mathrm{A}$. The distribution of the points can be characterized by the average slope and the scatter amplitude about the mean locus. The simulations indicate that these parameters depend on the dispersion of the magnetic field (mainly) and on the dispersion of the initial rotation period, in agreement with the supposition by Lyne et al. (1975). The importance of the dispersion of the initial field value was checked by performing simulations under the assumption that all pulsars are born with the same magnetic field. In this case, the dispersion of the initial periods only is not enough to explain the observed trend. If the dispersion of the initial period distribution is increased, the simulated data are more scattered, but the

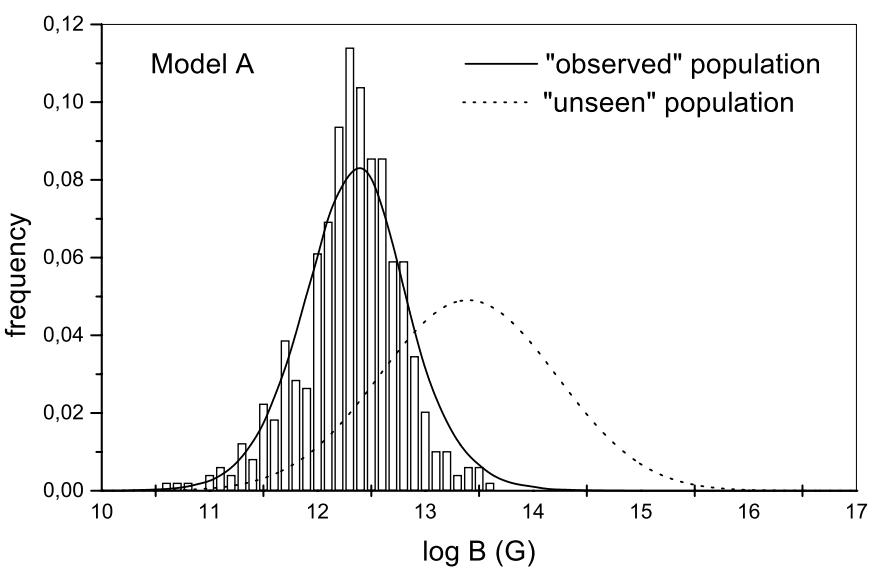

(a)

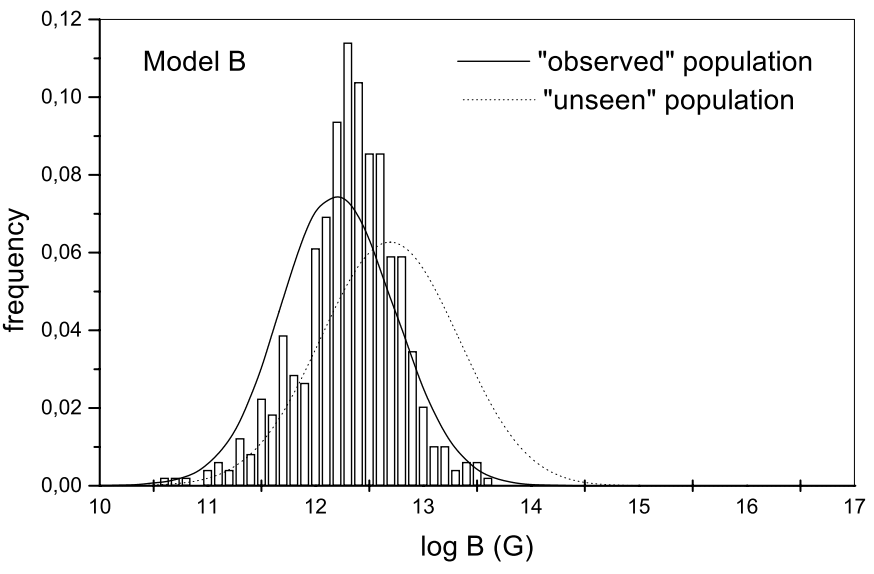

(b)

Fig. 4. a) Comparison between simulated (model A) magnetic field distributions (real and detected populations) and data (histogram). b) Same as Fig. 4a for model B.

slope cannot be reproduced. In this case, models including magnetic dissipation improve the results but always with a poorest fit quality in comparison with model $\mathrm{A}$.

Another interesting point concerns the comparison between the simulated magnetic field distribution of the observed and of the real ("unseen") pulsar populations. These distributions are displayed in Figs. 4a and 4b for models A and B respectively. The average field derived for the observed population is $2.5 \times 10^{12} \mathrm{G}$ for model $\mathrm{A}$ and $1.6 \times 10^{12} \mathrm{G}$ for model $\mathrm{B}$, in good agreement with the values obtained from the canonical model and data, but the fit quality for model $\mathrm{A}$ is better. As already noticed by other authors (see, for instance, Bhattacharya et al. 1992) the average magnetic field of the real population is higher than that of the observed one, and this tendency can clearly be seen in Figs. $4 \mathrm{a}$ and $4 \mathrm{~b}$. In the case of model $\mathrm{B}$, the average field of the unseen pulsars is about three times higher, whereas model $\mathrm{A}$ generates a population with an average field one order of magnitude higher than that of the detected pulsars. Such an effect 


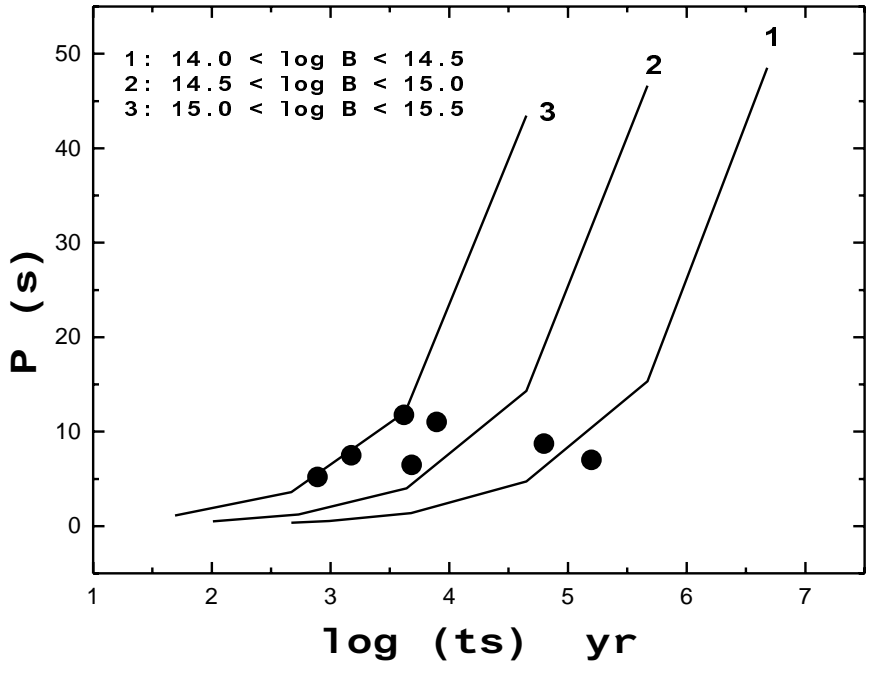

Fig. 5. Solid cuves correspond to average periods as a function of average indicative ages for different field strengths, derived from simulations. Circles correspond to data on magnetar candidates.

can be explained by the following reasons: firstly, pulsars generated at birth in the high side tail of the magnetic field distribution evolve rapidly to long periods. Consequently, their radio-luminosity decreases quite fast and the detection probability becomes rather small. Secondly, according to the beaming model by Biggs (1990), adopted in the present simulations, as period increases, the fraction of the sky covered by the pulsar emission cone decreases, reducing also the detection probability of those high field or long period objects. The latter case is very well illustrated by the radio pulsar J2144-3933 (Young et al. 1999), which has a period of $8.51 \mathrm{~s}$ and a very narrow pulse profile, with a half intensity of about one degree of longitude.

The statistics of the high field $\left(B>10^{14} \mathrm{G}\right)$ population are interesting to examine in some detail, in order to verify if they are compatible with the observed properties of AXPs and of SGRs. We report here essentially the results derived from model A, since from our point of view, they describe more adequately the high field population.

The pulsar birth rate derived from model $\mathrm{A}$ is one object born each 170 yr. Since the high field objects correspond to about $23 \%$ of the total population, their birth rate corresponds to about one object born each $750 \mathrm{yr}$.

From our simulations, the number of high field objects with rotation period less than $P$ is approximately given by $N(<P) \approx 0.85 P^{1.85}$. This equation predicts that the number of high field objects in the Galaxy with $P \leq 11 \mathrm{~s}$ is about 72 . Most of these objects have magnetic field strengths in the range $14.0<\log B<15.5$ and no higher field objects are expected to be detected in this period interval, since their number becomes appreciable only for periods longer than $130 \mathrm{~s}$. The present detected number of AXPs and SGRs with $P \leq 11$ s is about a dozen, suggesting that only a small fraction $(\approx 15 \%)$ of these objects have been discovered. These numbers must be taken with caution, since the selection effects on the detection of SGRs and AXPs are rather different of those present in pulsar surveys, simulated in our numerical experiments.

Seven objects suspected to be magnetars have their period derivative measured. For these objects, the indicative age $t_{\mathrm{s}}=P /(2 \dot{P})$ can be derived and a comparison with simulated data is possible. However, for young objects, $t_{\mathrm{s}}$ may overestimate the true age, depending on the initial period and on the initial field strength. Therefore, the simulated objects were classed into different bins of age and magnetic field, and their average properties, like period and indicative age, were computed after $10^{5}$ runs. In Fig. 5, the mean period is plotted against the mean indicative age and each curve corresponds to a given magnetic field interval. The available data (summarized in Table 2) are consistent with the statistical properties of the simulated population, supporting the hypothesis that AXPs and SGRs are neutron stars born in the high side tail of the magnetic field distribution.

\section{Discussion and conclusions}

Simulations performed by Bhattacharya et al. (1992), Hartman et al. (1997) and Mukherjee \& Kembhavi (1997) lead those authors to conclude that no significant field decay occurs $\left(t_{\mathrm{D}}>100-160 \mathrm{Myr}\right)$ during the active life time of the pulsar. The present simulations essentially confirm these results, since models without field decay are those which give the best representation of data. When the field decay is included in the pulsar evolution, we were unable to find a set of input parameters, characterizing the initial period and magnetic field distributions, which give a simultaneous acceptable representation of the observed period, first derivative and magnetic field distributions.

Our simulations indicate that the diagram $P \dot{P}-t_{\mathrm{s}}$ alone cannot be used to test the magnetic field decay without ambiguity. The dispersion of the values of the initial field and rotation period can explain by themselves the observed trend of the data points, namely, the average slope and scattering amplitude. Concerning the interpretation of that diagram, it is worth mentioning the following point: let us assume for a while that the field dissipation controls the distribution of the data points. Then, the best fit gives a relation of the form

$\log (P \dot{P})=a+b \cdot \log \left(t_{\mathrm{s}}\right)$

where $a=-3.86$ and $b=-0.78$ (c.g.s. units). This equation can immediately be integrated, resulting for the period evolution

$P \propto t^{(1+b) / 2} \propto t^{0.11}$.

This equation is equivalent to have assumed a power law for the magnetic field decay $\left(B \propto t^{b / 2} \propto t^{-0.39}\right)$ in the equation of motion. As a consequence of field decay, the pulsar deceleration is smaller than that predicted by the standard model and, in this case, the expected braking index $N=\frac{\ddot{\Omega} \Omega}{\Omega^{2}}$ is equal to $\frac{(3+b)}{(1+b)} \approx 10$, almost triple the canonical value $(N=3)$. Four young pulsars 
Table 2. Magnetar candidades.

\begin{tabular}{lcccc}
\hline Object & $P(\mathrm{~s})$ & $\log (\dot{P})$ & $\log \left(t_{\mathrm{s}}\right)(\mathrm{yr})$ & references \\
\hline SGR 1806-20 & 7.47 & -10.081 & 3.176 & Kouveliotou et al. (1998) \\
1E 1841-045 & 11.77 & -10.328 & 3.620 & Vasisht \& Gotthelf (1997) \\
1E 1048-59 & 6.45 & $-10.657:$ & 3.689 & Oosterbroek et al. (1998) \\
4U 0142+61 & 8.68 & -11.638 & 4.800 & Mereghetti \& Stella (1995) \\
1E 2259+58 & 6.98 & -12.136 & 5.202 & Mereghetti \& Stella (1995) \\
SGR 1900+14 & 5.16 & -9.958 & 2.893 & Kouveliotou et al. (1999) \\
1RX J1708-40 & 11 & -10.632 & 3.895 & Israel et al. (1999) \\
\hline
\end{tabular}

with measured braking indices have $N<3$ and, according to the scenario discussed in Paper I, these objects are probably in a phase where the magnetic dipole is still migrating. A relatively high magnetic field $\left(B \approx 4 \times 10^{13} \mathrm{G}\right)$ pulsar (J1119-6127) recently discovered (Kaspi et al. 1999) has a normal braking index $(N=3)$, suggesting that no direct evidence for the magnetic field decay has been observed until now in the period evolution.

The expected number of high field $\left(B>10^{14} \mathrm{G}\right)$ pulsars resulting from model $\mathrm{A}$ is about $23 \%$ of the total population and about 72 objects being members of this class, having periods less than $11 \mathrm{~s}$, are expected to be present in the Galaxy. The number of these objects predicted by model $\mathrm{B}$ is rather modest, of the order of $5-6$. Since a dozen magnetar candidates have already been discovered, this is another argument in favor of model A, which also predicts for the high field population relations between the mean period and mean indicative age compatible with the present available data on these objects. These statistical properties are not in contradiction to the interpretation that magnetars are objects born in the high side tail of the magnetic field distribution.

If this scenario is correct, why are AXPs radio quiet? Pair production by the mechanism $\gamma \rightarrow \mathrm{e}^{+} \mathrm{e}^{-}$is a necessary condition invoked in most pulsar emission models (Sturrock 1971). However, for fields in excess of the quantum critical field $B_{\mathrm{cr}}=\frac{m^{2} c^{3}}{e \hbar}=4.41 \times 10^{13} \mathrm{G}$, Baring \& Harding (1998) argued that photon splitting $(\gamma \rightarrow \gamma \gamma)$ becomes dominant, suppressing pair creation and the magnetosphere required for radio emission. The discovery of radio pulsars with magnetic fields comparable to the critical value (Kaspi et al. 1999; Camilo et al. 2000) argues against such a simple explanation. The photon splitting mechanism alone is not able to explain why two young pulsars $\left(t_{\mathrm{s}}<2000 \mathrm{yr}\right)$, J1119-6127 (Kaspi et al. 1999) and J1846-0258 (Gotthelf et al. 2000) having quite similar periods $(P=0.407 \mathrm{~s}$ and $0.323 \mathrm{~s}$ respectively) and magnetic fields ( $B=0.93 B_{\text {cr }}$ and $1.13 B_{\text {cr }}$ respectively) behave so dissimilarly. The former is a radio pulsar whereas the latter is a X-ray pulsar, associated with the supernova remnant Kes 75 . No radio emission has been observed from J1846-0258, but the object is probably located inside the central core of the SNR, a synchrotron plerion similar to the Crab, rendering difficult such a detection. More recently, Zhang \& Harding (2000) suggested that besides photon splitting, the orientation of the magnetic dipole with respect to the spin axis may also play an important role to establish the existence or not of radio emission, but this question still requires a more satisfactory answer.

The energy source of the observed X-rays is another problem to be faced if such an emission is not fed by field dissipation. A possible issue was considered by Heyl \& Hernquist (1997), who showed that for $B>10^{12} \mathrm{G}$, the quantization of the electron energies enhances the conductivity along the field lines. As a consequence, there is a net increase in the heat flux and high field objects may have effective temperatures up to $40 \%$ larger than low field neutron stars. Thus, enhanced cooling induced by a strong magnetic field could be a possible explanation for the X-ray emission observed in AXPs.

In conclusion, the magnetic field strength seems to play a major role in the evolution of young neutron stars. Objects born with $B \leq 10^{13} \mathrm{G}$ evolve as radio-loud pulsars, since curvature radiation produces a pair magnetosphere required for most emission models. In higher field objects $\left(B>10^{14} \mathrm{G}\right)$, the quantum process of photon splitting acts as a quenching mechanism for pairs, suppressing radio emission. In these stars, the high field also enhances the cooling and produces higher luminosities as well as temperature variations across the surface, which can explain the observed pulsed emission of AXPs. Three objects are presently known in the transition region $10^{13}<B<10^{14} \mathrm{G}$ and, as mentioned above, the understanding of their properties requires a considerable improvement of the existing models.

\section{References}

Baring, M. G., \& Harding, A. K. 1998, ApJ, 507, L55

Bhattacharya, D. 1990, in Neutron Stars: Theory and Observations, ed. J. Ventura, \& D. Pines, NATO ASI Ser., 344,103

Bhattacharya, D., Wijers, R. A. M. J., Hartman, J. W., \& Verbunt, F. 1992, A\&A, 254, 198

Biggs, J. D. 1990, MNRAS, 245, 514

Camilo, F., Kaspi, V. M., Lyne, A. G., et al. 2000 [astro-ph/0004330]

Cline, T., Frederiks, D. D., Golenetskii, S., et al. 2000, ApJ, 531,407

Colpi, M., Geppert, U., \& Page, D. 2000, ApJ, 529, L29

Epstein, R. I., \& Link, B. 2000 [astro-ph/0001365]

Goldreich, P., \& Reisenegger, A. 1992, ApJ, 395, 250 
Gotthelf, E. V., Vasisht, G., Boylan-Kolchin, M., \& Torii, K. Lyne, A. G., Ritchings, R. T., \& Smith, F. G. 1975, MNRAS, 2000 [astro-ph/0008097]

Gunn, J. E., \& Ostriker, J. P. 1970, ApJ, 160, 979

Hansen, B. M., \& Phinney, E. S. 1997, MNRAS, 291, 569

Hartman, J. W., Bhattacharya, D., Wijers, R., \& Verbunt, F. 1997, A\&A, 322, 477

Heyl, J. S., \& Hernquist, L. 1997, ApJ, 489, L67

Heyl, J. S., \& Kulkarni, S. R. 1998, ApJ, 506, L61 171,579

Lyne, A. G., \& Lorimer, D. R. 1994, Nature, 369, 127

Mereghetti, S. 1995, ApJ, 455, 598

Israel, G. L., Covino, S., Stella, L., et al. 1999, ApJ, 518, L107

Jones, P. B. 1976, Ap\&SS, 45, 369

Mereghetti, S. 1999 [astro-ph/9911252]

Mereghetti, S., \& Stella, L. 1995, ApJ, 442, L17

Mukherjee, S., \& Kembhavi, A. 1997, ApJ, 489, 928

Narayan, R., \& Ostriker, J. P. 1990, ApJ, 352, 222

Kaspi, V. M., Camilo, F., Lyne, A. G., et al. 1999 [astro-ph/9912281]

Kaspi, V. M., Lackey, J. R., \& Chakbrarty, D. 2000, ApJ, 537, L31

Oosterbroek, T., Parmar, A. N., Mereghetti, S., \& Israel, G. L. 1998, A\&A, 334, 925

Regimbau, T., \& de Freitas Pacheco, J. A. 2000, A\&A, 359, 242, Paper I

Sturrock, P. A. 1971, ApJ, 164, 529

Tauris, T. M., \& Manchester, R. N. 1998, MNRAS, 298, 625

Kouveliotou, C., Fishman, G. J., Meegan, C. A., et al. 1994, Nature, 368, 125

Taylor, J. H., Manchester, R. N., \& Lyne, A. G. 1993, ApJS, 88,529

Kouveliotou, C., Dieters, S., Strohmayer, T., et al. 1998, Nature, 393, 235

Kouveliotou, C., Strohmayer, T., Hurley, K., et al. 1999, ApJ, 510, L115

Link, B., Franco, L. M., \& Epstein, R. I. 1998, ApJ, 508, 838

Lorimer, D. R., Bailes, M., Dewey, R. J., \& Harrison, P. A. 1993, MNRAS, 263, 403

Lyne, A. G., Anderson, B., \& Salter, M. J. 1982, MNRAS, 201, 503

Thompson, C., \& Duncan, R. C. 1995, MNRAS, 275, 255

Thompson, C., \& Duncan, R. C. 1996, ApJ, 473, 322

Urpin, V., \& Konenkov, D. 1997, MNRAS, 292, 167

Vasisht, G., \& Gotthelf, E. V. 1997, ApJ, 486, L129

Young, M. D., Manchester, R. N., \& Johnston, S. 1999, Nature, 400,848

Zhang, B., \& Harding, A. K. 2000, ApJ, 535, L51 